\title{
Physical-Mathematical Model of Optical Radiation Interaction with Biological Tissues
}

\author{
Tetyana I. Kozlovska, Peter F. Kolisnik, Sergey M. Zlepko, Natalia V. Titova, Volodymyr S. Pavlov, Waldemar \\ Wójcik, Zbigniew Omiotek, Miergul Kozhambardiyeva, Aizhan Zhanpeisova
}

\begin{abstract}
The physical-mathematical model of distribution of optical radiation in biological tissues was developed. It allows determining the change of intensity of optical radiation depending on such parameters as installation angle of the sensor, biological tissue thickness and the wavelength. Were Obtained graphics which represent changes of the optical radiation intensity that is registered by photodetector depending on installation angle of the sensor, biological tissue thickness and the extinction coefficient

Keywords: photoplethysmography, biological tissue, skin, absorption, scattering, propagation, optical radiation, light beam.
\end{abstract}

\section{Introduction}

Human skin is a complex multilayer medium that contains blood vessels, through which is passed continuous blood flow. The main factors that affect on the distribution of laser radiation in biological tissues are the skin color, the presence of inhomogeneities and hairy. And all of them complicate the research of the processes of interaction of laser radiation with human skin. There are many mathematical and physical models that allow representing and describing of these processes and each of them is focusing on a specific case of studying [1].

The mathematical description of the light scattering and absorption processes can be made in two ways: by using analytical theory based on Maxwell's equations, and using transport theory. The main disadvantage of analytic theory is complexity of getting the accurate analytical solutions. Transport theory describes the interaction of laser radiation with tissue, and considers the transfer of photons by scattering and absorbing medium, without using Maxwell's equations [1, 3].

\section{Method}

Due to development of methods of laser diagnostics were expanded opportunities for studying of peripheral blood circulation. The use of lasers is based on the light interaction with biological tissues [1, 2], such as scattering, reflection and absorption. These processes depend on the pigmentation of the skin, blood composition and collagen fibers structure that determine the photons distribution in investigated area of the tissue. The absorption coefficient of the skin is mainly determined by the water and such pigments as melanin, bilirubin, $\beta$-carotene and hemoglobin [7]. Melanin is the base pigment and it is the main epidermal chromophor. The absorption coefficient of the epidermis and the corneal layer is determined by the melanin absorption. In the Figure 1 is represented absorption spectrum of melanin in the epidermal layer of human skin $[9,10]$.

The one of important value is the optical density (OD) which is calculated as:

$$
\mathrm{OD}=\mu_{m e l} \cdot h_{e}
$$

where $\mu_{m e l}$ - the coefficient of the absorption of melanin, $\mathrm{h}_{\mathrm{e}}$ - the thickness of the epidermis layer [1]

The amount of melanin depends on the type of skin and can be changed from $1,5 \%$ to $43 \%$.

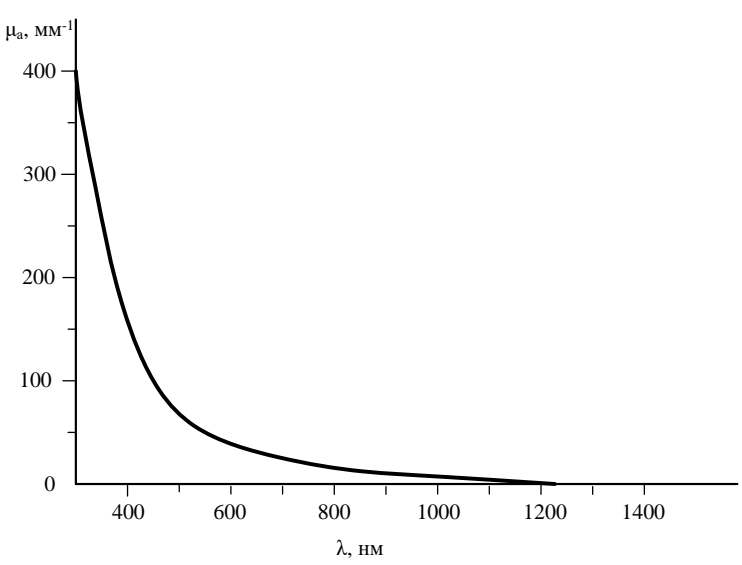

Fig. 1 Absorption spectrum of melanin $[9,10]$

The dermis structure of the skin is different from the epidermis structure and its scattering is stronger on the shorter wavelengths. As the penetration depth of radiation in dermis at different wavelengths depends on the scattering, penetration of longer wavelengths is more deeper than shorter. This is due to presence of melanin, which is contained in dermis and it absorbs shorter wavelengths. Propagation of the optical radiation in the different skin layers is shown in Fig. 2. [4].

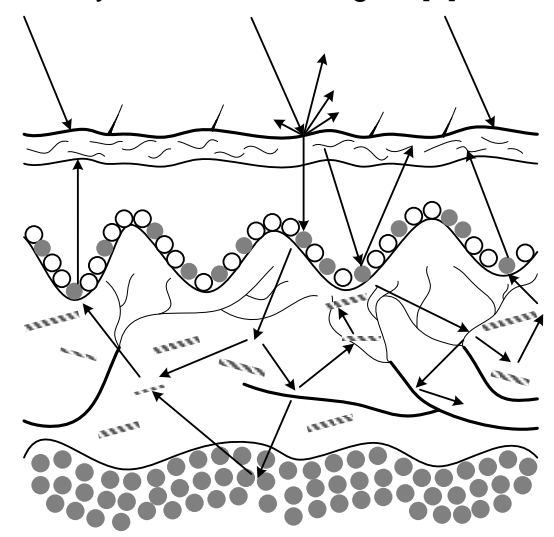

Fig. 2 Propagation of the optical radiation in the human skin [4]

The light that irradiates the skin area, takes different paths in its layers. Part of the incident radiation is reflected from the skin surface because there is refractive index difference in the corneal layer and the air and it is called as total internal reflection. Remain, is not reflected radiation from the skin surface (about 93-95\%), passes into the epidermis layer. In the epidermal layer radiation is almost not scattered but is absorbed by melanin. When the radiation passes through the dermal layer, is 
occurred the multiple scattering by collagen fibers and absorption by melanin.

The peak absorption of hemoglobin occurs on 280 , 420,540 and $580 \mathrm{~nm}$ with threshold wavelengths 600 $\mathrm{nm}$. The main feature of such molecules as hemoglobin and melanin is their complex threshold structure is located in 400-600 nm range. But in the near infrared region their absorption is strongly attenuated. The most deeply radiation passes into the tissues on the therapeutic window region $(600-1300 \mathrm{~nm})$ as a result of weak absorption and strong scattering. On the wavelengths over $1500 \mathrm{~nm}$ the water is the main absorption center [6].

In the Fig. 3 is represented dependence of the coefficient of the absorption and the depth of penetration of laser radiation on the biological tissue properties. As we can see from the figure the maximum depth of penetration is in therapeutic window region. That is why diode lasers, radiation of which is in the red and near infrared region is used for photoplethysmographic investigations.

By the electromagnetic field elastic-charged particles start moving. When the oscillation frequency of particles coincides with the frequency of the waves is a significant absorption due to resonance. When the frequencies of particles do not coincide with the wave frequencies, radiation is scattered. One of the reasons of the scattering is inhomogeneity of the refractive index at the microscopic level $[2,11]$.

As the skin is strongly scattered medium, coherent photons can be multiple scattered, that leads to a large angular deviation of radiation relative to the initial direction of the beam. Secondary radiation is incoherent, broadband and unpolarized. Besides it, secondary laser radiation provides greater depth of penetration into biological tissues because its degree of attenuation for each centimeter of tissue depth is almost 10 times less than primary $[2,11]$.

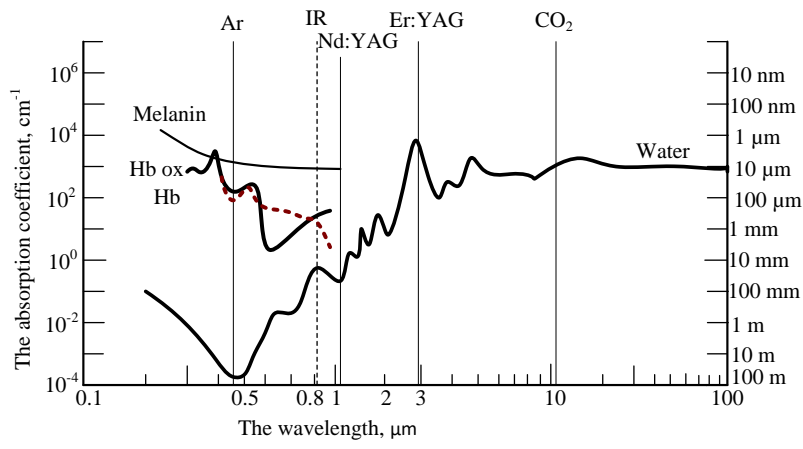

Fig. 3 Dependence of the absorption coefficient and the depth of penetration of the water, hemoglobin and melanin on the wavelengths

After multiple scattering some photons can go back from the tissue under a random angle that creates diffusely reflected light. In the case when photon is repeatedly scattered, probability of the back scattering is significantly increased. It depends on the wavelengths of the initial radiation and can be about $30-40 \%$ of the incident beam. Thus components that go back on the surface of the tissue as a result of back scattering are informative and they can determine metabolic, physiological or structural condition of the tissues. The main property of scattering tissue is anisotropy that is determined as g-anisotropy factor [11].

For the most of non-transparent biological tissues anisotropy factor values are in the range $(0.7 \div 0.95)$. Dependence of the anisotropy factor of the skin (dermis and epidermis) can be described by the following empirical relation $[9,11]$

$$
g(\lambda)=0,7645+0,2355 \cdot\left[1-\exp \left(\frac{\lambda-500 \mathrm{~nm}}{729.1 \mathrm{~nm}}\right)\right] .
$$

Dependence of the scattering coefficient of the skin on the wavelength is described $[9,11]$

$$
\mu_{S}(\lambda)=\mu_{S}\left(\lambda_{0}\right) \cdot\left(\frac{\lambda}{700 \mathrm{~nm}}\right)^{-3 / 2}
$$

The absorption and scattering of photons in tissues are characterized by the absorption coefficient $\mu \mathrm{a}$ and the scattering coefficient $\mu \mathrm{s}$. They show attenuation of the incident beam intensity as a result of absorption and scattering and depend on the photon path length in the investigation tissue. Back-scattered photons that go back on the tissue surface allow analysing and reproducing images of internal structure of the tissues. It allows detecting of pathological changes in the tissues, including tumors.

\section{Results}

The intensity of the optical radiation that is registered by the sensor photodetector depends on the following parameters as installation angle of the sensor, biological tissue thickness and wavelength. And it can be determined such way

$$
\mathrm{I}=\mathrm{I}_{0}-\left(\mathrm{I}_{\text {loss.sens. }}+\mathrm{I}_{\text {loss.scat. }}+\mathrm{I}_{\text {loss.abs. }}\right),
$$

where $I_{0}$ - is the intensity of the incident radiation on the biological tissue;

$\mathrm{I}_{\text {loss.sens. }}$ - loss of radiation intensity depending on installation angle of the sensor;

$$
\mathrm{I}_{\text {loss.scat. }} \text { - loss of radiation intensity by the scattering; }
$$

$\mathrm{I}_{\text {loss.abs. }}$ - loss of radiation intensity by the absorption.

The loss of radiation intensity depending on installation angle of the sensor on the investigated biological tissue is determined as [2]

$$
I_{\text {loss.sens. }}=I_{0}-I_{\text {detect. }} \text {, }
$$

where

$$
\mathrm{I}_{\text {detect. }}=\mathrm{I}_{0} \cdot \frac{\tau_{\text {opt. }} \cdot \pi \cdot \mathrm{r}^{2}}{\mathrm{~L}^{2}} \cdot \cos \alpha \cdot \cos \beta,
$$

where $\mathrm{I}_{0}$ - the intensity of the incident radiation on the biological tissue; $\left(\mathrm{I}_{0}=\frac{\mathrm{J}}{l^{2}}\right.$, where $\mathrm{J}-$ is candle power, $l$ - distance between the light source and the surface of biological tissue);

$\tau_{\text {opt }}$ - coefficient that characterizes the light passage through the optical system $(0,9-0,95)$;

$\mathrm{L}$ - distance between biological tissue and photodetector; $\alpha$ - angle of the incident light on the photodetector area;

$\beta$ - the angle between the normal to a surface and an incoming light ray.

Loss of the radiation intensity by the scattering is determined 


$$
\mathrm{I}_{\text {loss.scat. }}=\mathrm{I}_{0}-\mathrm{I}_{\text {scat. }} \text {. }
$$

According to the Lambert law $[1,2,5,10]$

$$
\mathrm{I}_{\text {scat. }}=\mathrm{I}_{0} \cdot \exp \left(-\mu_{\mathrm{s}} \cdot \mathrm{z}\right)
$$

where $\mu_{\mathrm{s}}$-scattering coefficient of the biological tissue, $\mathrm{cm}^{-1}$;

$\mathrm{z}-$ thickness of the biological tissue, $\mathrm{m}$.

The scattering coefficient depending on the wavelength is determined

$$
\mu_{\mathrm{s}}=\mu_{\mathrm{s}_{1}}(\lambda)+\mu_{\mathrm{s}_{2}}(\lambda)+\ldots+\mu_{\mathrm{s}_{\mathrm{n}}}(\lambda),
$$

where $\mu_{s_{1}}(\lambda) \cdots \mu_{s_{n}}(\lambda)$ - scattering coefficients of the optical radiation for skin layers ( $\mathrm{n}$ - number of layers); $\lambda$ - wavelength.

Equation (7) with (8) and (9) have the following form

(10) $I_{\text {scat. }}=I_{0} \cdot\left[1-\exp \left[-\left(\mu_{s_{1}}(\lambda)+\mu_{s_{2}}(\lambda)+\ldots+\mu_{s_{n}}(\lambda)\right) \cdot z\right]\right]$.

Loss of the radiation intensity by the absorption is determined

$$
\mathrm{I}_{\text {loss.abs. }}=\mathrm{I}_{0}-\mathrm{I}_{\text {abs. }} .
$$

Iabs. is determined [1, 2, 9-11]

$$
I_{\text {abs. }}=I_{0} \cdot \exp \left(-\mu_{\mathrm{a}} \cdot \mathrm{z}\right),
$$

where $\mu_{\mathrm{a}}$ - absorption coefficient of the biological tissue, $\mathrm{cm}^{-1}$.

Depending on the wavelength the absorption coefficient is determined as sum of absorption coefficients of each layer of biological tissue

$$
\mu_{a}=\mu_{a 1}(\lambda)+\mu_{a 2}(\lambda)+\ldots+\mu_{a \mathrm{n}}(\lambda),
$$

where $\mu_{a 1}(\lambda) \ldots \mu_{a \mathrm{n}}(\lambda)$ - absorption coefficients of different skin layers.

Equation (11) with (12) and (13) have the following form

(14) $I_{\text {loss.abs }}=I_{0} \cdot\left\lfloor 1-\exp \left[-\left(\mu_{a_{1}}(\lambda)+\mu_{a_{2}}(\lambda)+\ldots+\mu_{a_{n}}(\lambda)\right) \cdot z\right\rfloor\right.$.

Equation (4) with (2), (6), (10), (14) and some transformations have the following form

$$
\begin{aligned}
& \text { 15) } \mathrm{I}=\mathrm{I}_{0} \cdot\left(1-\left(\left(1-\frac{\tau_{\text {opt. }} \cdot \pi \cdot \mathrm{r}^{2}}{\mathrm{~L}^{2}} \cdot \cos \alpha \cdot \cos \beta\right)+\right.\right. \\
& \left.\left.+\left[1-\exp \left[-\left(\mu_{t_{1}}(\lambda)+\mu_{t_{2}}(\lambda)+\ldots+\mu_{t_{n}}(\lambda)\right) \cdot z\right]\right]\right)\right),
\end{aligned}
$$

where $\mu_{t_{1}}(\lambda) \ldots \mu_{t_{n}}(\lambda)$ - extinction coefficients for different skin layers, where $\mu_{t}(\lambda)=\mu_{s}(\lambda)+\mu_{a}(\lambda)$.

As a result were obtained graphics and represented changes of the optical radiation intensity, that is registered by photodetector depending on installation angle of the sensor, biological tissue thickness and the extinction coefficient (Fig. 4, 5).

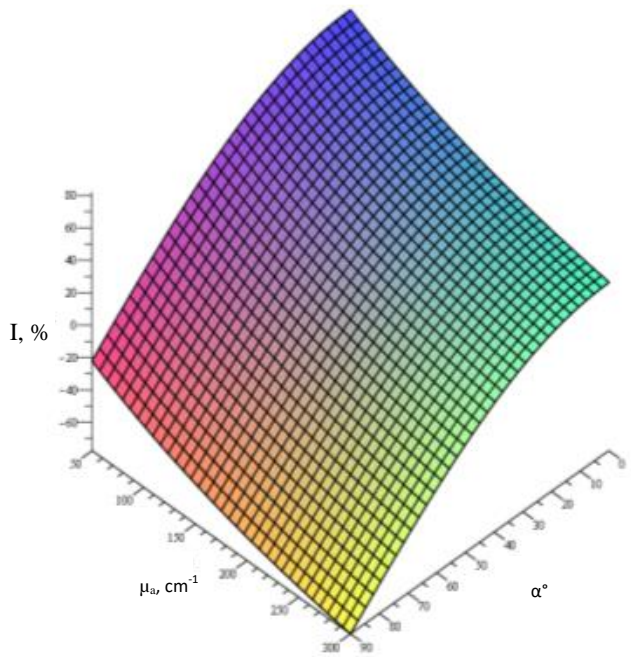

Fig. 4 Dependence of the optical radiation intensity that is registered by photodetector on installation angle of the sensor and the extinction coefficient

I, \%

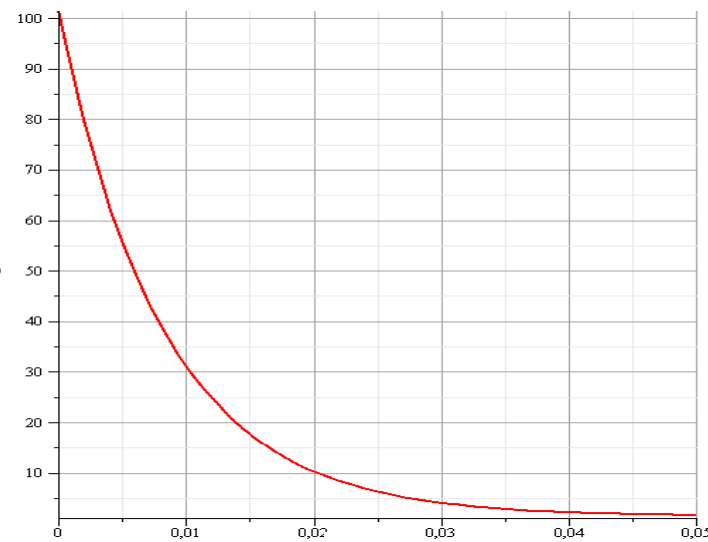

$\mathrm{d}, \mathrm{m}$

Fig. 5 Dependence of the optical radiation intensity that is registered by photodetector on the biological tissue thickness

\section{Conclusions}

The developed physical-mathematical model of investigation of optical radiation propagation in biological tissues allow to research how radiation is attenuated depending on such parameters as installation angle of the sensor, biological tissue thickness and the wavelength.

Authors: Doctor of Engineering Sciences, professor, Vicerector for scientific work, Sergiy V. Pavlov, Vinnytsia National Technical University, Khmelnytsky Hwy, 95, 21021 Vinnitsa, Ukraine, E-mail: psv@vntu.edu.ua.

Candidate of Engineering Sciences, Tetyana I. Kozlovska, Vinnytsia National Technical University, Khmelnytsky Hwy, 95, 21021 Vinnitsa, Ukraine, E-mail: t.i.kozlovska@gmail.com.

\section{REFERENCES}

[1] Pushkareva A. I. Methods of mathematical modeling in optics of biological tissues: tutorial. / Pushkareva A. I - SPb: SPbSU ITMO, 2008. - 103 p. 
[2] Physical principles of biomedical optics: monograph / [S.V. Pavlov, V.P. Kozhemiako, P.F. Kolesnik et al.]. - Vinnytsya: VNTU, 2010. - $152 \mathrm{p}$.

[3] Photoplethysmohrafic technologies of the cardiovascular control / [Pavlov S.V., Kozhemiako V.P., Petruk V.G., Kolesnik P.F.]. Vinnitsa: Universum-Vinnitsa, 2007. - 254 p.

[4] A simulation model of distribution of optical radiation in biological tissues / S.V. Pavlov, S.E. Tuzhanskyy, T.I. Kozlovska, A.V. Kozak // Visnyk VNTU. - 2011. - №3. - P. 191-195.

[5] Deryabin E.I. Application of photoplethysmohraphic method to studying of local blood flow of maxillofacial region / E.I. Deryabin., E.E. Dvinyaninova, V.G. Vaganova [et al.] // Laser Medicine. - № 3 (2). - 1999. - P. 72-26.

[6] Allen J. Photoplethysmography and its application in clinical physiological measurements / Allen J. // Physiol. Meas. - Vol. 28. - 2007. - P. 21-39.

[7] Kubelka P. Ein beitrag zur optik der farbanstriche Zurich Tech. / P. Kubelka, F. Munk. - Physik, 1931. - P. 543.

[8] Prahl S. A. Determining the optical properties of turbid media using the adding-doubling method / S. A. Prahl, M. C. van Gemert and A. J. Welch. // Applied Optics. - 1993. - № 32(4). - P. 559568.

[9] Sardar D. K. Optical properties of whole blood / D. K. Sardar and L. B. Levy // Lasers in Medical Science. - 1998. - P. 106-111.

[10] G. Yoon. Absorption and Scattering of Laser Light in Biological Media - Mathematical Modeling and Methods for Determining Optical Properties. PhD thesis, University of Texas at Austin, USA. - 1988. - P. 45.

[11]Optical biomedical diagnostics [6 vols.]. - Moscow: FYZMATLYT, 2007. - [Trans. with English. under. Ed. V. Tuchyn]. T. 1 - 2007. - 560 p 